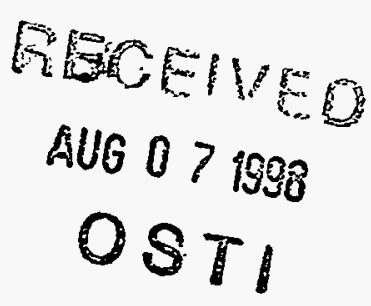

\title{
Radionuclide Concentrations in Honey Bees from Area G at TA-54 during 1997
}

DISTRIBUTION OF THIS DOCUMENT IS URLLMITED
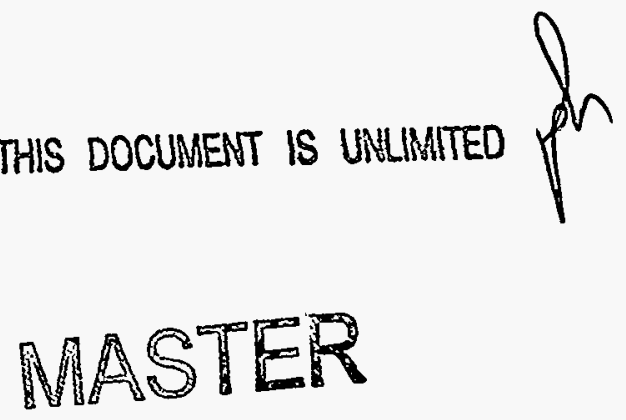

\section{LOS Alamos}

Los Alamos National Laboratory is operated by the University of California for the United States Department of Energy under contract W-7405-ENG-36. 


\section{Edited by Hector Hinojosa, Group CIC-1}

This is the first report in this series.

An Affirmative Action/Equal Opportunity Employer

This report was prepared as an account of work sponsored by an agency of the United States Government. Neither The Regents of the University of Califormia, the United States

Government nor any agency thereof, nor any of their employees, makes any warranty, express or implied, or assumes any legal liability or responsibility for the accuracy, completeness, or usefulness of any information, apparatus, product, or process disclosed, or represents that its use would not infringe privately owned rights. Reference herein to any specific commercial product, process, or seroice by trade name, trademark, manufacturer, or otherwise, does not necessarily constitute or imply its endorsement, recommendation, or favoring by The Regents of the University of Colifornia, the United States Government, or any agency thereof. The views and opinions of authors expressed herein do not necessarily state or reflect those of The Regents of the University of California, the United States Government, or any agency thereof. Los Alamos National Laboratory strongly supports academic freedom and a researcher's right to publish; as an institution, however, the Laboratory does not endorse the viewpoint of a publication or guarantee its tecinical correctness. 


\section{DISCLAIMER}

Portions of this document may be illegible electronic image products. Images are produced from the best available original document. 
LA-13480-PR

Progress Report

UC-2000

Issued: July 1998

Radionuclide Concentrations in Honey Bees

from Area G at TA-54 during 1997

T. K. Haarmann

P. R. Fresquez 


\title{
RADIONUCLIDE CONCENTRATIONS IN HONEY BEES \\ FROM AREA G AT TA-54 DURING 1997
}

\author{
T.K. Haarmann and P.R. Fresquez
}

\begin{abstract}
Honey bees were collected from two colonies located at Los Alamos National Laboratory's Area G, Technical Area 54, and from one control (background) colony located near Jemez Springs, NM. Samples were analyzed for the following: $\operatorname{cesium}\left({ }^{137} \mathrm{Cs}\right)$, americium $\left({ }^{241} \mathrm{Am}\right)$, plutonium $\left({ }^{238} \mathrm{Pu}\right.$ and $\left.{ }^{239,240} \mathrm{Pu}\right)$, tritium $\left({ }^{3} \mathrm{H}\right)$, total uranium, and gross gamma activity. Area $\mathbf{G}$ sample results from both colonies were higher than the upper (95\%) level background concentration for ${ }^{238} \mathrm{Pu}$ and ${ }^{3} \mathrm{H}$.
\end{abstract}

\section{INTRODUCTION}

As part of the ongoing environmental surveillance program at Area G (Fresquez et al. 1997a) - a 25.5ha $(63-a c)$ low-level radioactive waste management and disposal area located on the east end of Mesa del Buey at Technical Area (TA) 54 at Los Alamos National Laboratory (LANL) (Figure 1)-samples of honey bees were collected from beehives during the summer of 1997. Honey bees can be thought of as mobile samplers that efficiently cover a large sample area and then return to a central location (Bromenshenk 1992). Honey bees forage in an area with a radius as large as $6 \mathrm{~km}(3.7 \mathrm{mi})$ and often cover a total area up to 100 square $\mathrm{km}$ (39 square mi) Leita et al. 1996, Visscher and Seeley 1982). Each hive contains literally thousands of bees, most of whom will forage for nectar, water, pollen, and plant resins, which are all brought back into the hive. During these foraging flights, bees inadvertently contact and accumulate a wide array of pollutants, 


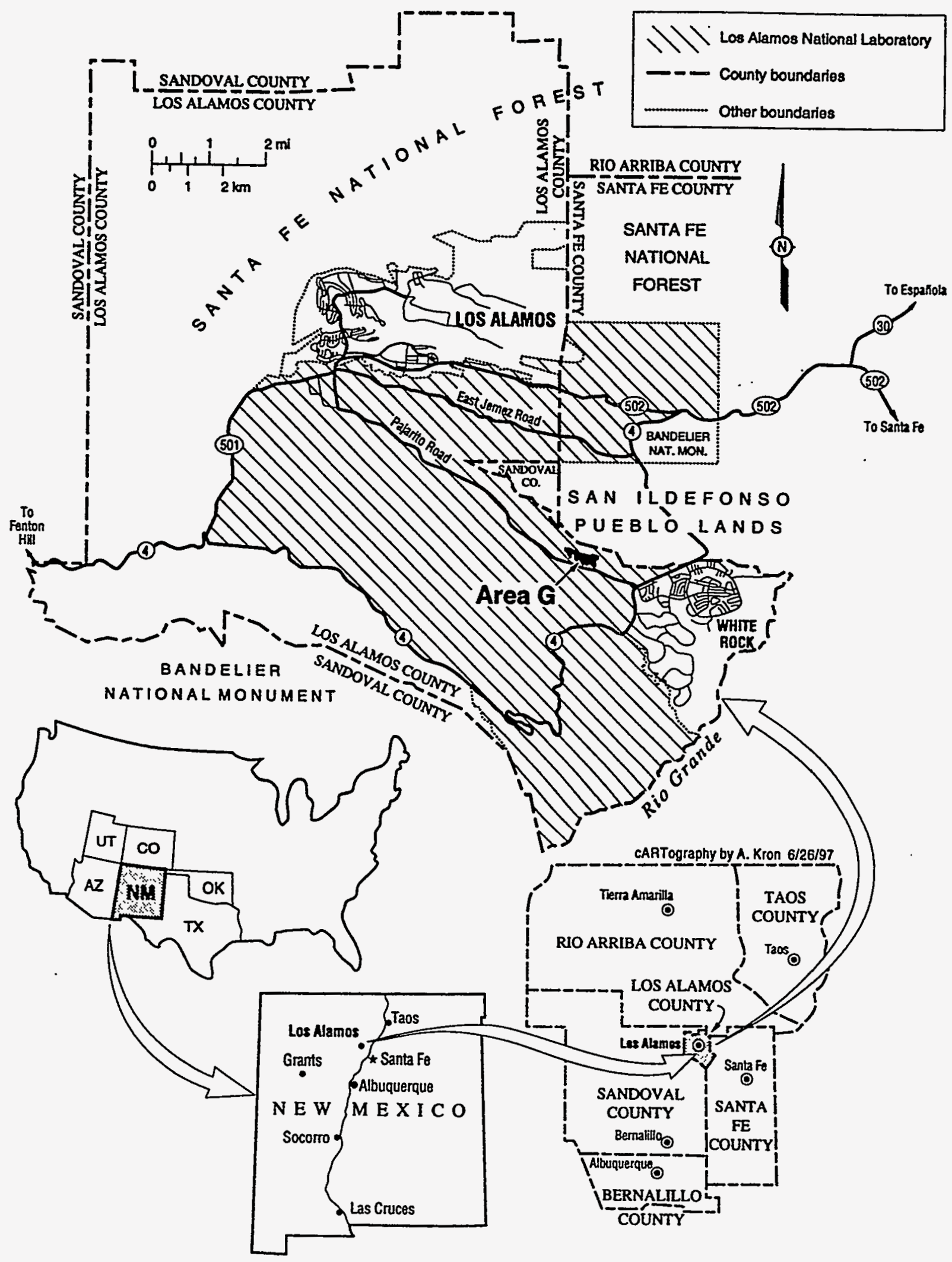

Figure 1. The location of Area G at Los Alamos National Laboratory. 
some of which are brought back to the colony (Bromenshenk et al. 1985). These contaminants often become incorporated into the bee tissue, the wax, the honey, or the hive itself (WallworkBarber et al. 1982).

Honey bee studies have been conducted on many different types of pollutants including fluoride (Bromenshenk et al. 1988a, Mayer et al. 1988), lead (Migula et al. 1989), zinc (Bromenshenk et al. 1988b), nickel (Balestra et al. 1992), potassium (Barbattini et al. 1991), cesium (Bettoli et al. 1987, Tonelli et al. 1990), tritium (White et al. 1983, Fresquez et al. 1997b), and plutonium (Hakonson and Bostick 1976). It is an inexpensive form of monitoring, especially considering the many different sampling points the foraging bees visit. Collection of bees at one location (the hive) can provide a plethora of information from numerous points concerning the distribution and bioavailability of contaminants. Comparing the amounts of contaminants in honey bees with the known amounts of contaminants in the surrounding area could be useful for modeling the redistribution of contaminants through ecosystems. The very nature of honey bee ecology makes them an excellent living system from which to monitor the presence of contaminants.

The objective of this study was to compare various radionuclide concentrations in honey bees from Area $\mathrm{G}$ with honey bees collected from a background location.

\section{METHODS}

We monitored Area G using beehives consisting of a standard Langstroth hive stocked with Italian honey bees (Apis mellifera ligustica). During 1997, two colonies were established on the south end of Area G near the ${ }^{3} \mathrm{H}$ shafts (Figure 2). These colonies were brought into the study site from an uncontaminated area. In addition, a control (background) site with one colony was established $10 \mathrm{~km}$ ( $6 \mathrm{mi})$ south of Jemez Springs, NM.

After three months, bee tissue samples were collected from all of the colonies. Three separate samples (one from each colony), each containing approximately $100 \mathrm{~g}$ of bees, were collected. Each individual $100-\mathrm{g}$ sample consisted of approximately 1,000 bees. 


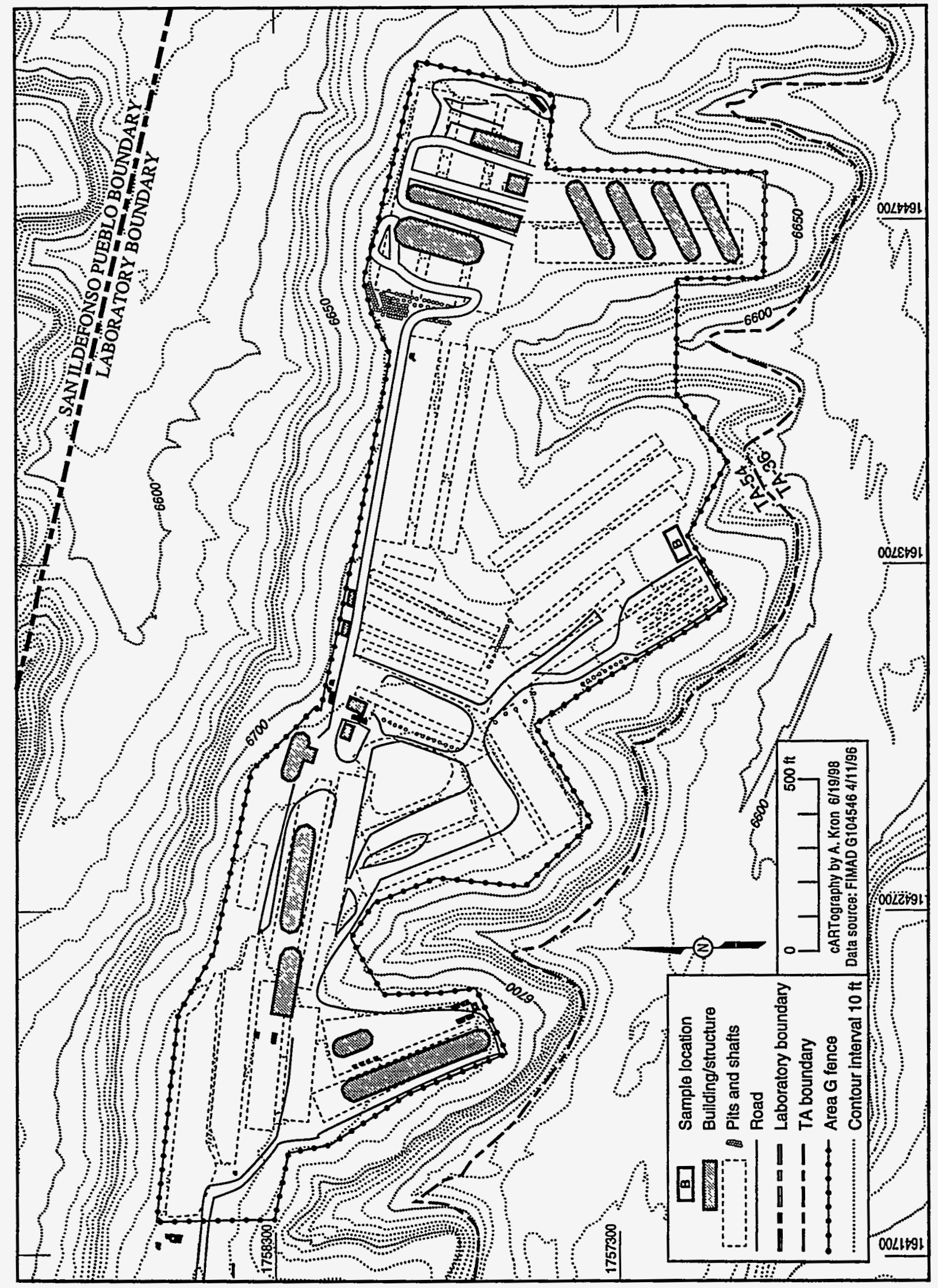

Figure 2. Site/sample location of bee hives at Area G. 
Bee samples were collected using a small, rechargeable vacuum. Bees were vacuumed off frames that were removed from the honey supers, transferred to a plastic resealable bag, weighed, and double bagged into plastic resealable bags. All samples were kept in a cooler and frozen upon returning to the laboratory. With each sample collected, the vacuum collection area was thoroughly cleaned to avoid crosscontamination of samples.

All samples were analyzed by LANL's Environmental Chemistry Group for ${ }^{3} \mathrm{H},{ }^{137} \mathrm{Cs},{ }^{241} \mathrm{Am},{ }^{238} \mathrm{Pu}$, ${ }^{239,240} \mathrm{Pu}$, total uranium, and gamma activity. Analytical methods have been previously described in Fresquez et al. (1997a). The bee tritium samples were analyzed by liquid scintillation counting in the following manner: $5 \mathrm{ml}$ of moisture were distilled from each sample, mixed with $15 \mathrm{ml}$ of a scintillation solution, and counted on a scintillation counter for 50 minutes. All gamma-emitting radionuclide concentrations were determined using highresolution germanium detector gammaray spectrometry. $\mathrm{Pu}$ and $\mathrm{Am}$ samples were dissolved in nitric acid, isolated by anion exchange, electroplated onto stainless steel disks and counted using an alpha spectrometer. Total uranium was determined by kinetic phosphorescence analysis.

\section{RESULTS}

Table 1 contains a summary of the analytical results from samples collected near Area $G$ and the control site. The original analytical reports are included in Appendix A for future reference. In general, most radionuclides, with the exception of ${ }^{238} \mathrm{Pu}$ and ${ }^{3} \mathrm{H}$, were within or just above the regional statistical reference level (RSRL). The RSRL is the upper (95\%) level background concentration (mean + two std dev) from the present data. And, of these two radionuclides $\left({ }^{238} \mathrm{Pu}\right.$ and ${ }^{3} \mathrm{H}$ ), only ${ }^{3} \mathrm{H}$ concentrations were at detectable levels-where the analytical result was higher than two times the counting uncertainty. Tritium levels in the Area $\mathrm{G}$ bees, for example, were at 82.8 and $110.20 \mathrm{pCi} \mathrm{mL}^{-1}$; the control colony contained only $1.03 \mathrm{pCi} \mathrm{mL}^{-1}$. These data are consistent with other surveillance studies of ${ }^{3} \mathrm{H}$ (and other radionuclides) at Area $\mathrm{G}$ in bees 
(Fresquez et al. 1997b), soils (Conrad et al. 1996, Fresquez et al. 1997c), small mammals (Biggs et al. 1997), and vegetation (Fresquez et al. 1997c).

\section{ACKNOWLEDGMENT}

Thanks to Rebecca J. Wechsler, Environmental Coordinator, EM-SWO, for technical assistance at Area G.

Table 1. Analytical Results from Honey Bee Samples Collected from Colonies at Area $\mathbf{G}$ and a Control Site in 1997.

\begin{tabular}{|c|c|c|c|c|c|c|c|c|}
\hline $\begin{array}{l}\text { Element/ } \\
\text { Activity }\end{array}$ & Units & $\begin{array}{c}\text { Area G } \\
\text { C-1 }\end{array}$ & $\mathbf{A U}^{\mathrm{a}}$ & $\begin{array}{c}\text { Area G } \\
\text { C-2 }\end{array}$ & $\mathbf{A U}$ & Control & $\mathbf{A U}$ & RSRL $^{\mathbf{b}}$ \\
\hline${ }^{137} \mathrm{Cs}$ & $\mathrm{pCi} / \mathrm{g}^{\mathrm{c}}$ & 0.52 & 0.78 & 0.86 & 0.29 & -0.33 & 1.81 & 3.29 \\
\hline U-total & $\mu \mathrm{g} / \mathrm{g}^{\mathrm{c}}$ & 0.18 & 0.02 & 0.13 & 0.01 & 0.13 & 0.01 & 0.15 \\
\hline${ }^{241} \mathrm{Am}$ & $\mathrm{pCi} / \mathrm{g}^{\mathrm{c}}$ & 0.0329 & 0.0075 & 0.0234 & 0.0058 & 0.0165 & 0.0067 & 0.0299 \\
\hline${ }^{238} \mathrm{Pu}$ & $\mathrm{pCi} / \mathrm{g}^{\mathrm{c}}$ & 0.0026 & 0.0039 & 0.0021 & 0.0034 & -0.0089 & 0.0024 & -0.0041 \\
\hline${ }^{239,240} \mathbf{P u}$ & $\mathrm{pCi} / \mathrm{g}^{\mathrm{c}}$ & 0.0113 & 0.0060 & 0.0020 & 0.0027 & 0.0095 & 0.0050 & 0.0195 \\
\hline${ }^{3} \mathbf{H}$ & $\mathrm{pCi} / \mathrm{mL}^{\mathrm{d}}$ & 82.80 & 3.60 & 110.20 & 4.40 & 1.03 & 0.75 & 2.53 \\
\hline Gamma & $\mathrm{pCi} / \mathrm{g}^{\mathrm{c}}$ & 36.0 & 16.3 & 28.9 & 14.1 & 16.9 & 9.3 & 36.0 \\
\hline
\end{tabular}

${ }^{2}$ Analytical uncertainty; values are the uncertainty in the analytical results at the $65 \%$ confidence level (one sigma).

${ }^{b}$ Regional Statistical Reference Level; the upper (95\%) level background concentration (mean + two sigma) from present control data.

${ }^{\circ}$ Units are in $\mathrm{g}$ per ash.

${ }^{\mathrm{d}}$ Units are in $\mathrm{mL}$ tissue moisture.

\section{REFERENCES}

Balestra, V., G. Celli, and C. Porrini, "Bees, Honey, Larvae and Pollen in Biomonitoring of Atmospheric Pollution,” Aerobiologia 8:122-126 (1992).

Barbattini, R., F. Frilli, M. Iob, C. Giovani, and R. Padovani R, "Transfer of Cesium and Potassium by the "Apiarian Chain" in Some Areas of Friuli [NE Italy]," Apicoltura 7:8587 (1991). 
Biggs, J.R., K.D. Bennett, and P.R. Fresquez, "Radionuclide Contaminant Analysis of Small Mammals at Area G, Technical Area 54, 1996 (with cumulative summary for 1994-1996)," Los Alamos National Laboratory report LA-13345-MS (1997).

Bettoli, M.G., A.G. Sabatini, M.A. Vecchi, "Honey Produced in Italy since the Chernobyl Incident," Apitalia 14(10):5-7 (1987).

Bromenshenk, J.J., "Site-specific and Regional Monitoring with Honey Bees: Case Study Comparisons," In Proc. of Int. Symp. on Ecological Indicators, Volume 39, Fort Lauderdale, FL. 16-19 Oct. 1990, Elsevier Sci.Publ. London, UK (1992).

Bromenshenk, J.J., S.R. Carlson, J.C. Simpson, J.M. Thomas, "Pollution Monitoring of Puget Sound with Honey Bees," Science 227:800-801 (1985).

Bromenshenk, J.J., R.C. Cronn, J.J. Nugent, and G.J. Olbu, "Biomonitoring for the Idaho National Engineering Laboratory: Evaluation of Fluoride in Honey Bees," American Bee Journal 128(12):799-800 (1988a).

Bromenshhenk, J.J., J.L. Gudatis, R.C. Cronn, J.J. Nugent, and G.J. Olbu, "Uptake and Impact of Heavy Metals to Honey Bees," American Bee Journal 128(12):800-801 (1988b).

Conrad, R., M. Childs, C. Rivera-Dirks, and F. Coriz, "Area G Perimeter Surface-Soil and Single-Stage Water Sampling," Los Alamos National Laboratory report LA-13165PR (1996).

Fresquez, P.R., D.R. Armstrong, and L.H. Pratt, "Radionuclides in Bees and Honey Within and Around Los Alamos National Laboratory," Journal of Environmental Science and Health, A32(5) 1309-1323 (1997a). 
Fresquez, P.R., E.L. Vold, and L. Naranjo, "Radionuclide Concentrations in Soils and Vegetation at Radioactive-Waste Disposal Area G during 1996 Growing Season," Los Alamos National Laboratory report LA-13332-PR (1997b).

Fresquez, P.R., D.R. Armstrong, and L.H. Pratt, "Tritium Concentrations in Bees and Honey at Los Alamos National Laboratory: 1979-1996," Los Alamos National Laboratory report LA-13202-MS (1997c).

Hakonson, T.E., and K.V. Bostick, "The Availability of Environmental Radioactivity to Honey Bee Colonies at Los Alamos," Journal of Environmental Quality 5(3):307-309 (1976).

Leita, L., G. Muhlbachova, S. Cesco, R. Barbattini, C. Mondini, "Investigation of the Use of Honey Bees and Honey Bee Products to Assess Heavy Metals Contamination," Environmental Monitoring and Assessments 43:1-9 (1996).

Mayer, D.G., I.D. Lunden, L.H. Weinstein, "Evaluation of Fluoride Levels and Effects on honey Bees (Apis mellifera L.)," Fluoride 21:113-120 (1988).

Migula, P., K. Binkowska, A. Kafel, M. Nakonieczy, "Heavy Metal Contents and Adenylate Energy Charge in Insects from Industrialized Region as Indices of Environmental Stress," Proceedings of the 5th International Conference, Bioindicatores Deteriorisationis Regionis, II. Ceske Budejovice, Czechoslovakia: Institute of Landscape Ecology (1989).

Tonelli, D., E. Gattavecchia, S. Ghini, C. Porrini, G. Celli, A.M. Mercuri, "Honey Bees and their Products as Indicators of Environmental Radioactive Pollution," Journal of Radioanalytical and Nuclear Chemistry 141(2):427-436 (1990). 
Visscher, P.K., and T.D. Seeley, "Foraging Strategy of Honey Bee Colonies in a Temperate Deciduous Forest," Ecology 63:1790-801 (1982).

Wallwork-Barber, M.K., R.W. Ferenbaugh, and E.S. Gladney, "The Use of Honey Bees as Monitors of Environmental Pollution," American Bee Journal 122(11):770-772 (1982).

White, G.C., T.E. Hakonson, and K.V. Bostick, "Fitting a Model of Tritium Uptake by Honey Bees to Data," Ecological Modelling 18(3/4):241-251 (1983). 
APPENDIX A

CST ANALYTICAL REPORTS OF RADIONUCLIDES IN BEES 


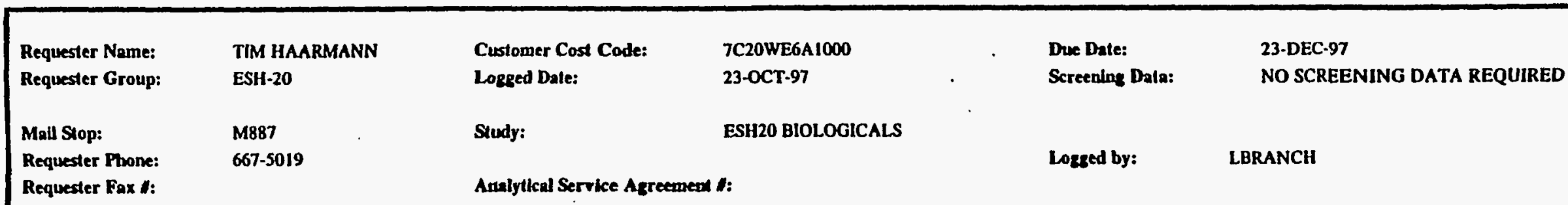

\section{CUSTOMER SAMPLES}

Method

GENERIC GAMMA

\begin{tabular}{|c|}
\hline$D$ \\
\hline
\end{tabular}

Sample Id

\section{3}

200043494

200043497
Task Id

300118357

300118366

300118386
Customer Id

Component

G1.97

G2.97

C1.97
CS-137

CS-137

CS-137
Result Value Uncertainty

0.78

1.81

0.86

$-0.33$
Units Qualifier
pCi/g

$\mathrm{pCi} / \mathrm{g}$ pCi/g

\section{DUPLICATE TASKS}

None run for this submission

SAMPLE SPIKES

None run for this submission 


\section{************ CST QUALITY ASSURANCE REPORT **********}

\section{BLIND QC}

None run for this submission

\section{METHOD BLANKS}

\begin{tabular}{|c|c|c|}
\hline Customer Id & Task Id & Component \\
\hline 00.22785 & 300143215 & CS.137 \\
\hline \multicolumn{3}{|l|}{ OPEN QC } \\
\hline Customer Id & Task Id & Component \\
\hline 00.33987 & 300143216 & CS-137 \\
\hline
\end{tabular}

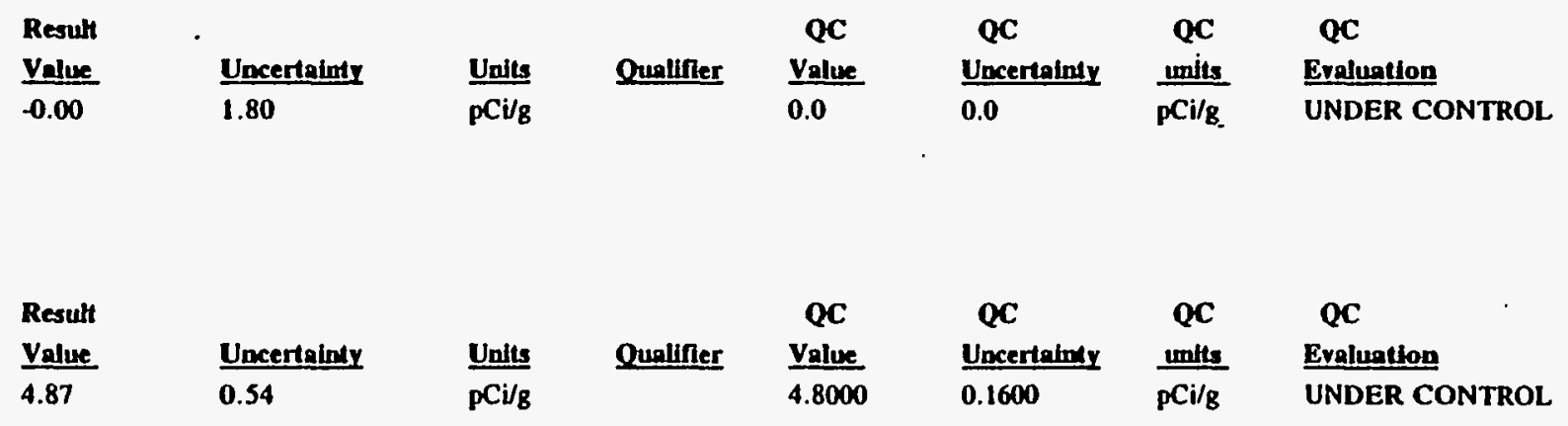



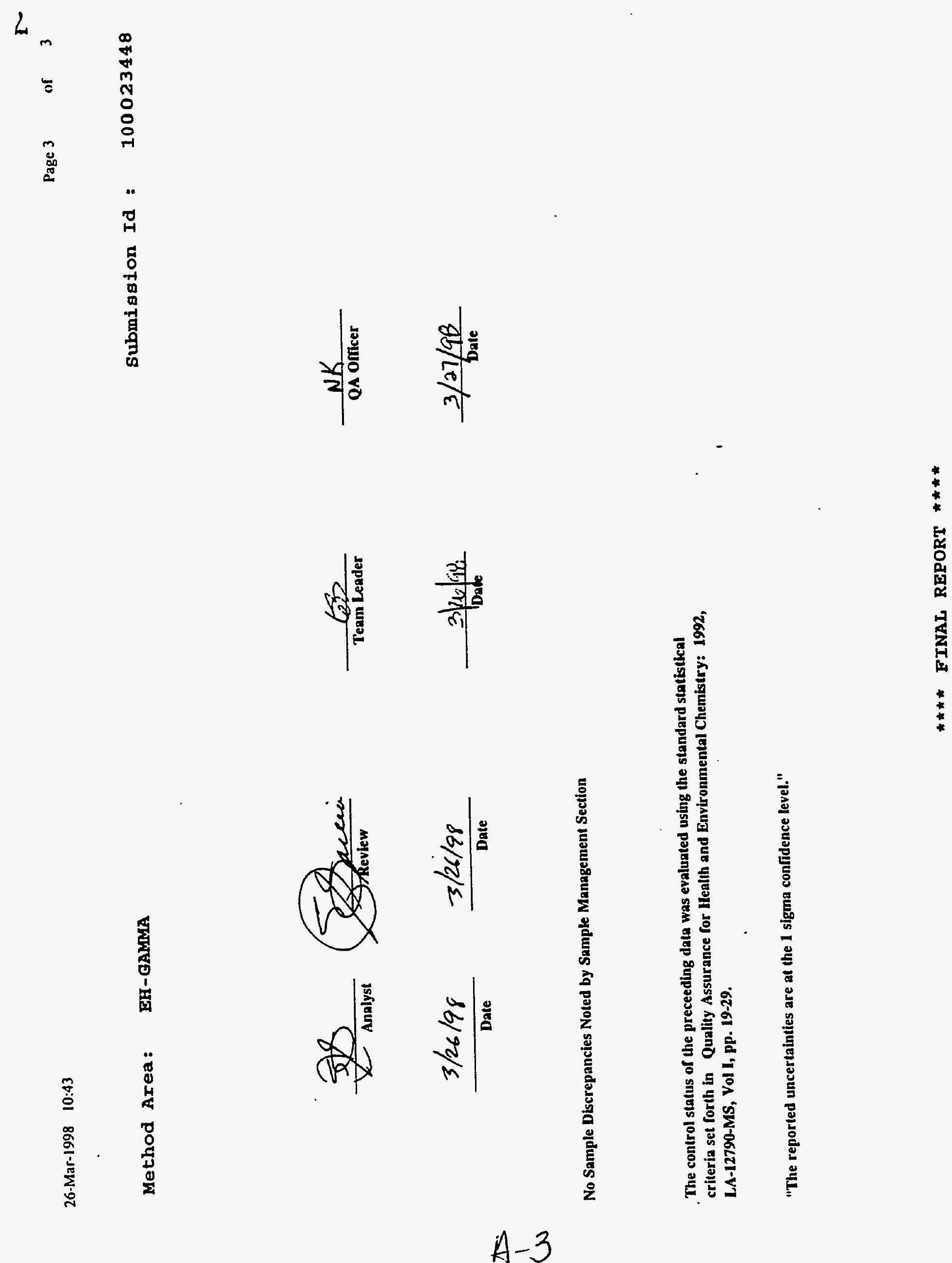


\begin{tabular}{|c|c|c|c|c|c|}
\hline Requester Name: & TIM HAARMANN & Customer Cost Code: & 7C20WE6A1000 & Due Date: & 23-DEC-97 \\
\hline Requester Group: & ESH.20 & Logged Date: & 23-0CT.97 & Screening Dala: & NO SCREENING DATA REQUIRED \\
\hline Mail Stop: & M887 & Study: & ESH20 BIOLOGICALS & & \\
\hline Requester Phone: & 667.5019 & & & Logged by: & LBRANCH \\
\hline Requester Fax \#: & & Analytical Service Agr & & & \\
\hline
\end{tabular}

\section{CUSTOMER SAMPLES}

Method

GENERIC KPA

Sample 1d

200043493

200043494

200043497

300118363
300118391

Customer Id

Component

G1-97

G2-97

C1.97

\section{U}

U

$\mathbf{u}$

\section{Result Value}

\subsection{8}

0.13

0.13

\section{DUPLICATE TASKS}

None run for this submission

\section{SAMPLE SPIKES}

None run for this submission 
81709

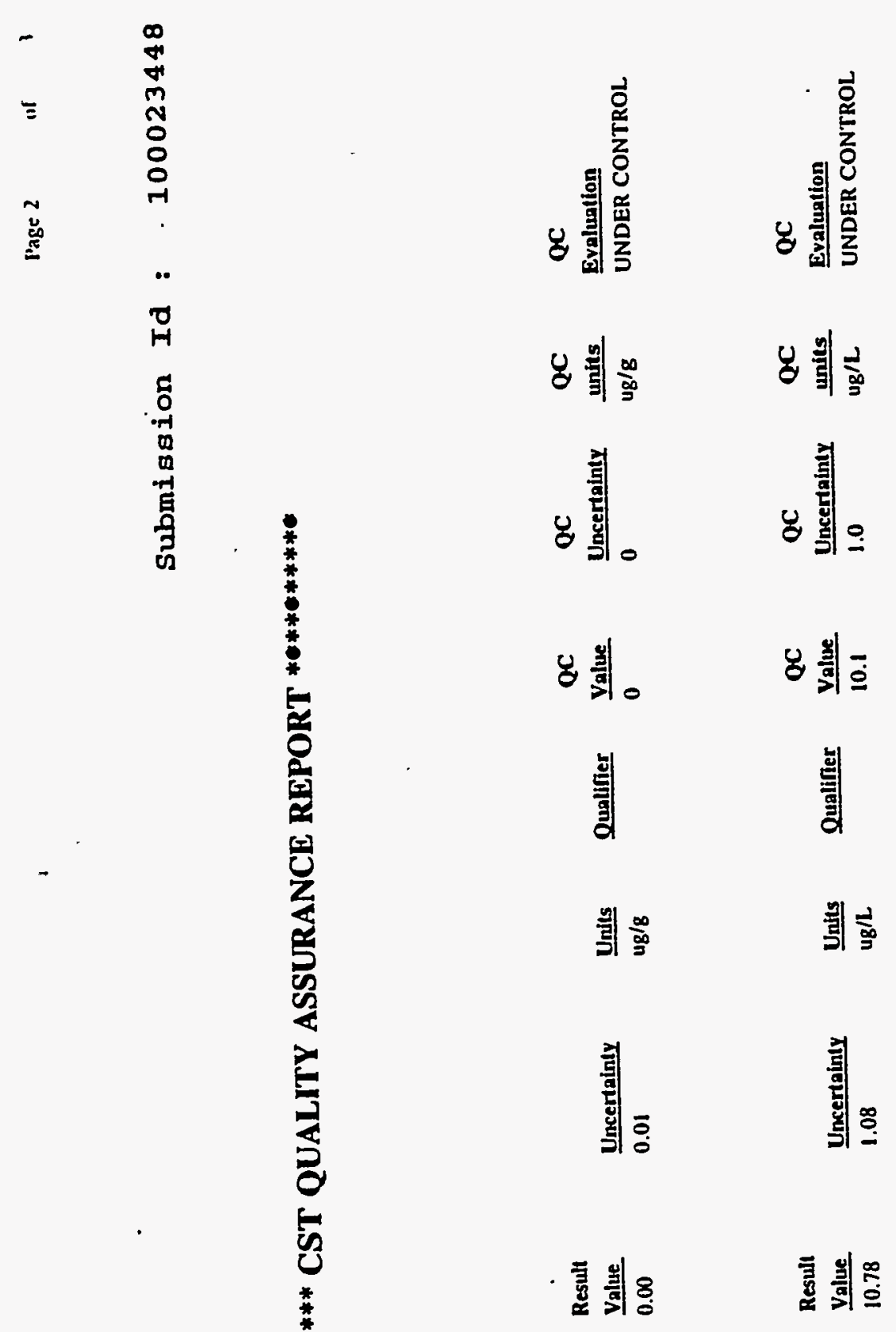

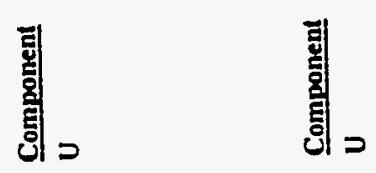




$$
\begin{aligned}
& \frac{\text { nl }}{\text { Analyst }} \quad \frac{\text { rgy }_{\text {Review }}}{\frac{3 / 31 / 98}{\text { Date }}} \quad \frac{4-1-98}{\text { Date }}
\end{aligned}
$$$$
\frac{63}{\text { Team Leader }}
$$

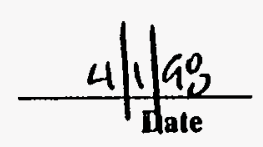




\begin{tabular}{|c|c|c|c|c|c|}
\hline Requesler Name: & TIM UAARMANN & Cuslomer Cosl Code: & 7C2OWEGALOOO & Due Date: & 23.DEC.97 \\
\hline Requester Group: & IESIH.20 & Logged Date: & $23.0 \mathrm{Cr}-97$ & Screening Data: & NO S(REENING DATA REQUIRFI) \\
\hline Mail Stop: & M887 & Study: & ESH20 BIOLOGICALS & & \\
\hline Requester Phone: & 667.5019 & & & Logged by: & LBRANCH \\
\hline Requester Fax H: & & Analytical Service AgI & & & \\
\hline
\end{tabular}

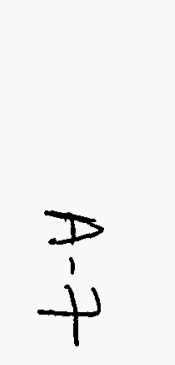

\section{CUSTOMER SAMPLES}

Method

AM RAS ENV

\begin{tabular}{|c|c|c|c|c|c|c|c|}
\hline Sample Id & Task Id & Customer Id & Component & Resull Value & Uncertainty & Units & Qualifier \\
\hline \multirow{10}{*}{200043493} & 300118359 & G1.97 & Am-241 & 0.0329 & 0.0075 & $\mathrm{pC}: \mathrm{i} / \mathrm{g}$ & \\
\hline & & G1.97 & Am-243T Spike & 1.95 & & $\mathrm{pCi}$ & \\
\hline & & G1-97 & Am-243T Recovery & 56.25 & & $\%$ & \\
\hline & & G1.97 & Am-243T Recovered & 1.1 & & $\mathrm{pCi}$ & \\
\hline & & G1.97 & Am-241 Gross Counts & 31 & & counts & \\
\hline & & G1.97 & Am.24I Background Counts & 6.2 & & counts & \\
\hline & & G1.97 & Efriciency & 19.94 & & $\%$ & \\
\hline & & (61.97 & Counn Time & 3000.00 & & $\min$ & \\
\hline & & G1.97 & Analysis Dale & 04/08/98 & & $\mathrm{MM} / \mathrm{DD} / \mathrm{Y}$ & \\
\hline & & G1.97 & Instrumeil & ALPHA 80 & & NONE & \\
\hline \multirow[t]{9}{*}{$200 \times(143494$} & 300118.368 & G2.97 & $A m \cdot 241$ & 0.0234 & 0.0058 & $\mathrm{pCi} / \mathrm{g}$ & \\
\hline & & G2.97 & Am-243'T Spike & 1.95 & & $\mathrm{pCi}$ & \\
\hline & & G2.97 & Am-243T Recovery & 58.33 & & $\%$ & \\
\hline & & - 62.97 & Am.243T Recovered & 1.14 & & $\mathrm{pCi}$ & \\
\hline & & G2.97 & Am-241 Gross Counis & 24 & & counts & \\
\hline & & 62.97 & Am-241 Background Counts & 3.8 & & counts & \\
\hline & & G2.97 & Erriciency & 19.02 & & $\%$ & \\
\hline & & G2.97 & Counu Time & 30100.00 & & $\min$ & \\
\hline & & (j2.97 & Analysis Date & $04 / 06 / 98$ & & $\mathrm{MM} / \mathrm{DD} / \mathrm{Y}$ & YY \\
\hline
\end{tabular}




\section{Method}

AM RAS ENV

\section{Sample Id}

200043494

\section{Task Id}

Customer Id

Component

Instrument

Am-241

Am-243T Spike

Am-243T Recovery

Am-243T Recovered

Am-241 Gross Counts

Am-241 Background Counts

Efficiency

Count Time

Analysiș Date

Insirument

Submission Id

\section{DUPLICATE TASKS}

None run for this submission

\section{SAMPIE SPIKES}

None run for this submission

C. 1.97

C1.97

C 1.97

C1 97

\section{Result Value Uncerlainty Unils Qualifier}

\section{ALPIIA 80}

0.0165

1.95

43.39

0.85

10

2.2

20.24

3000.00

04/08/98

ALPHA 80
NONE

$\mathrm{pCi} / \mathrm{g}$

pCi

$\%$

pCi

counts

counts

$\%$

$\min$

MM/DD/YY

NONE 

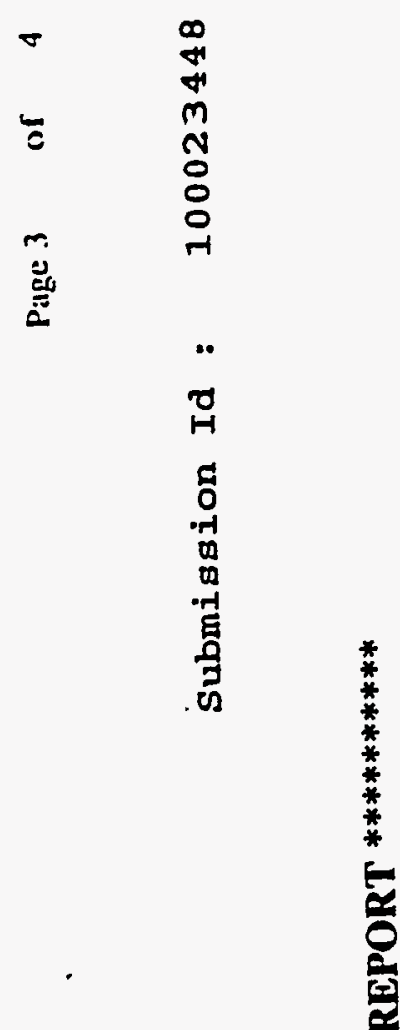

î.

造

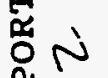

䰹

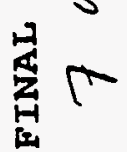

2

武

空

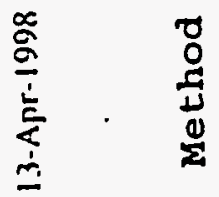

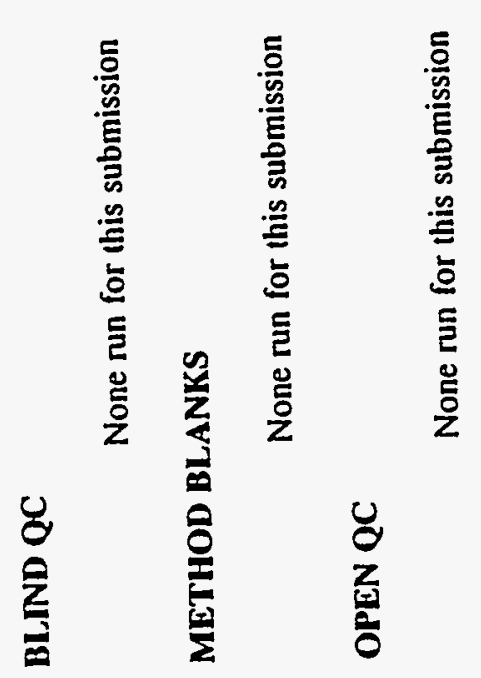

$A-9$ 

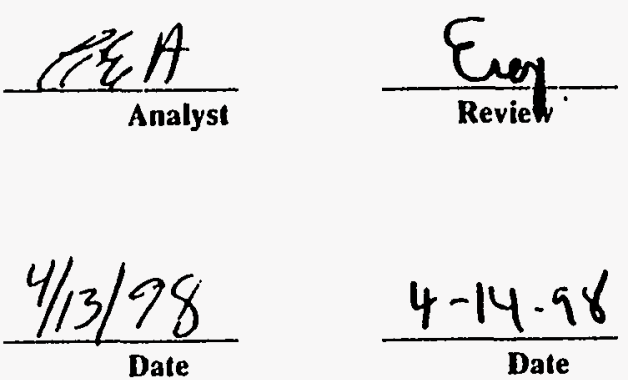
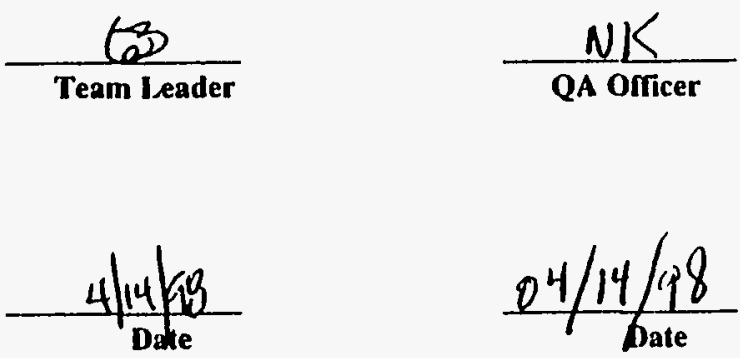

No Sample Discrepancies Noted by Sample Management Section

The control status of the preceeding data was evaluated using the standard statistical criteria set forth in Quality Assurance for Health and Environmental Chemistry: 1992, I.A-12790-MS, Vol I, pp. 19-29.

"The reported uncertainties are at the I sigma confidence level." 
Method Area: EH-ALPHA

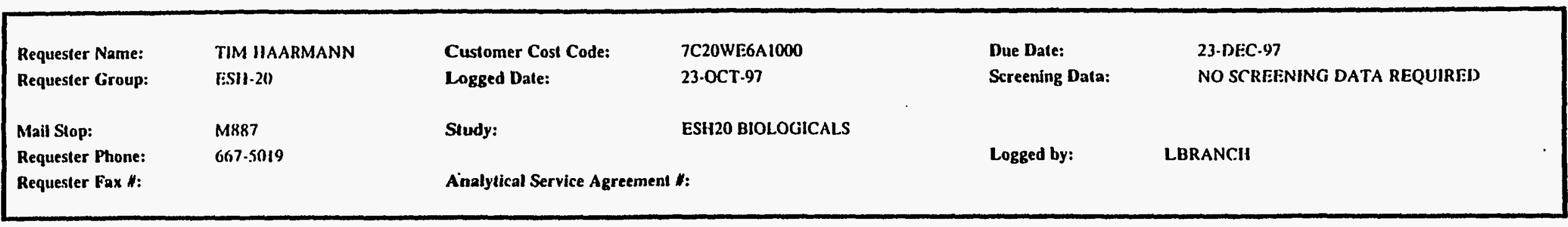

\section{CUSTOMER SAMPLES}

\section{Method}

PURAS ENV

\begin{tabular}{|c|c|c|c|c|c|c|}
\hline Sample Id & Task Id & Customer Id & Component & Result Value & Uncertainty & Qualifier \\
\hline & \multirow{9}{*}{300118358} & G1.97 & Pu-239 & 0.0113 & 0.0060 & $p C(i / g$ \\
\hline & & G1.97 & Pu-242T Recovery & 33.47 & & $\%$ \\
\hline & & G1.97 & Pu-242T Recovered & 0.65 & & $\mathrm{pCi}$ \\
\hline & & G1.97 & Pu-238 Gross Counts & 6 & & counts \\
\hline & & G1.97 & Pu-239 Background Counts & 7.2 & & counts \\
\hline & & G1 97 & Efficiency & 30.54 & & $\%$ \\
\hline & & G1.97 & Count Time & 3000.00 & & $\min$ \\
\hline & & G1.97 & Analysis Date & $04 / 2 / 98$ & & MM/DD/YY \\
\hline & & G1.97 & Instruntent & ALPHA 32 & & NONE: \\
\hline & \multirow{2}{*}{$3(x) 118367$} & 62.97 & Pu 242T Recovered & 0.91 & & $\rho \mathrm{C}_{1}$ \\
\hline & & 02.97 & Pu-2.38 Gross Counts & 10 & & counts \\
\hline
\end{tabular}


Method

PU RAS ENV

\begin{tabular}{|c|c|c|}
\hline Sample Id & Task Id & Customer \\
\hline \multirow[t]{7}{*}{$2(x)(1) 43494$} & 300118367 & G2.97 \\
\hline & & G2.97 \\
\hline & & G2.97 \\
\hline & & G2-97 \\
\hline & & G2-97 \\
\hline & & G2.97 \\
\hline & & G2.97 \\
\hline \multirow[t]{13}{*}{$20 K \times 14.3497$} & $3(k) 118387$ & C 1.97 \\
\hline & & $\mathrm{C} 1.97$ \\
\hline & & C. 1.97 \\
\hline & & C. 1.97 \\
\hline & & C.1.97 \\
\hline & & C.1.97 \\
\hline & & C) 97 \\
\hline & & $C 1.97$ \\
\hline & & C1.97 \\
\hline & & $C 1.97$ \\
\hline & & $C 1.97$ \\
\hline & & $C 1.97$ \\
\hline & & $C 1.97$ \\
\hline
\end{tabular}

\section{DUPLICATE TASKS}

None run for this submission

\begin{tabular}{|c|c|c|c|}
\hline Component & Resull Value & Uncertainty & $\underline{\text { Units }}$ \\
\hline Pu-238 Background Counts & 7.6 & & counts \\
\hline Pu-239 Gross Counts & 8 & & counts \\
\hline Pu-239 Background Counts & 5.8 & & counts \\
\hline Efficiency & 30.81 & & $\%$ \\
\hline Count Time & $3(001) .00$ & & $\min$ \\
\hline Analysis Date & $04 / 2 / 98$ & & $\mathrm{MM} / \mathrm{DD} / \mathrm{Y}$ \\
\hline Instrument & Al.PHA 32 & & NONE \\
\hline Pu-238 & .0 .01899 & $0 .(0) 24$ & $\mathrm{pCi} / \mathrm{g}$ \\
\hline Pu-239 & $0 .(x) 95$ & $0 .(0150)$ & $\mathrm{pC} / \mathrm{g}$ \\
\hline Pu-242T Spike & 1.95 & & $\mathrm{pCi}$ \\
\hline Pu-242T Recovery & 4020 & & $\%$ \\
\hline Pu-242T Recovered & 0.78 & & $\mathrm{pC} i \mathrm{i}$ \\
\hline Pu-238 Gross Counts & 2 & & counts \\
\hline Pu-238 Background Counts & 8.0 & & counts \\
\hline Pu.239 Gross Cousus & 11 & & conumis \\
\hline Pu-239 Background Counts & 46 & & counts \\
\hline Effriciency & 31.12 & & $\%$ \\
\hline Count Time & $30 \times(10.000$ & & $\operatorname{mın}$ \\
\hline Analysis Date & $04 / 2 / 98$ & & $M M / D D / Y$ \\
\hline Instrument & ALIPHA 32 & & NONE \\
\hline
\end{tabular}


Method Area: EH-ALPHA

SAMPLE SPIKES

None run for this submission 

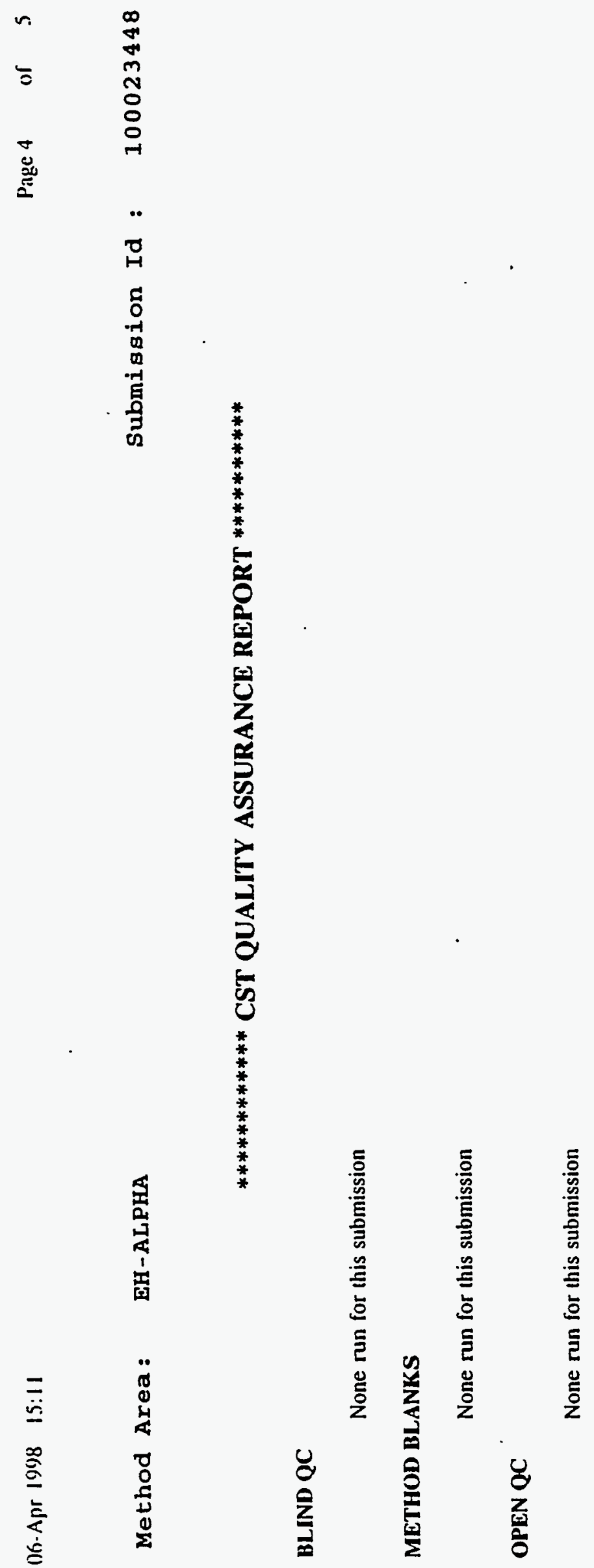

$A-14$ 

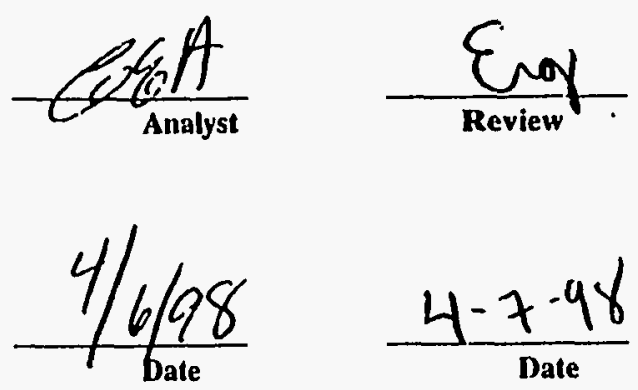
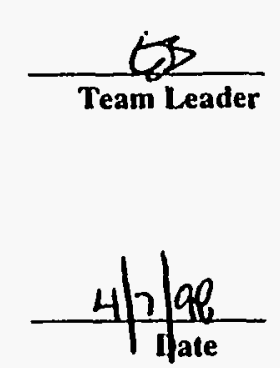

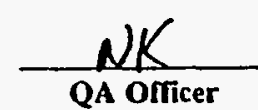

$\frac{04 / 07 / 98}{\text { bate }}$

No Sample Discrepancies Noted by Sample Management Section

The control status of the preceeding data was evaluated using the standard statistical criteria set forth in Quality Assurance for Health and Environmental Chemistry: 1992, I.A-12790-MS, Vol I, pp. 19-29.

"The reported uncertainties are at the 1 sigma confidence level." 


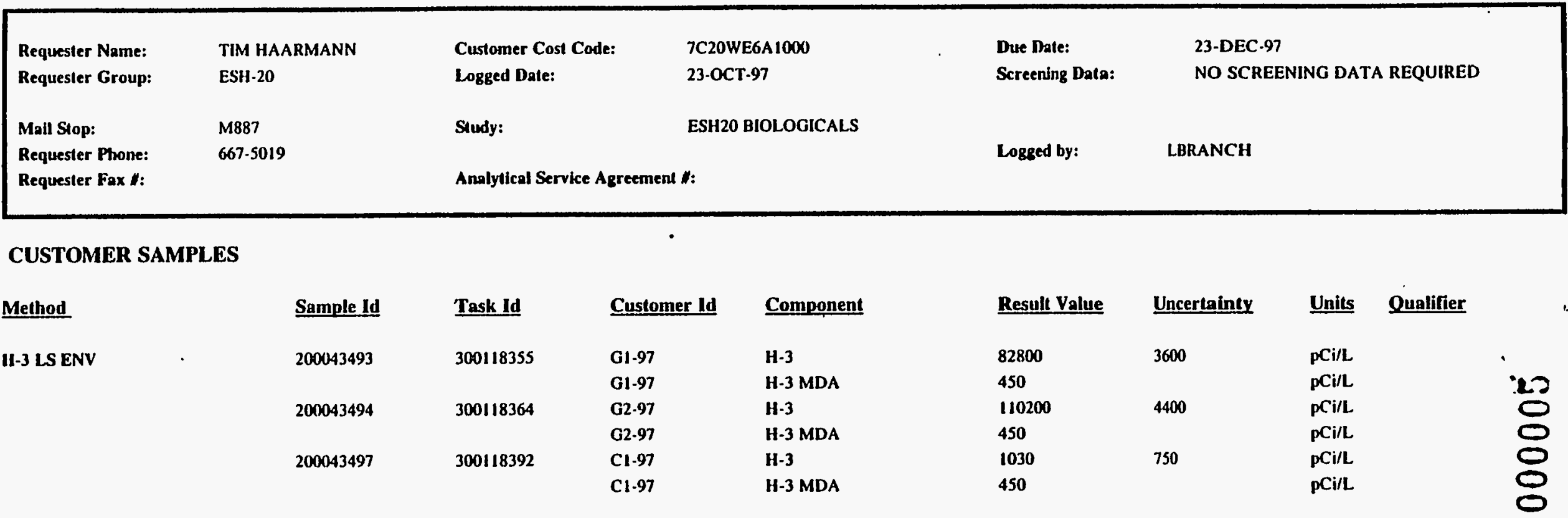

\section{DUPLICATE TASKS}

None run for this submission

\section{SAMPLE SPIKES}

None run for this submission 


\section{BLIND QC}

None run for this submission

\section{METHOD BLANKS}

None run for this submission

OPEN QC

None run for this submission 


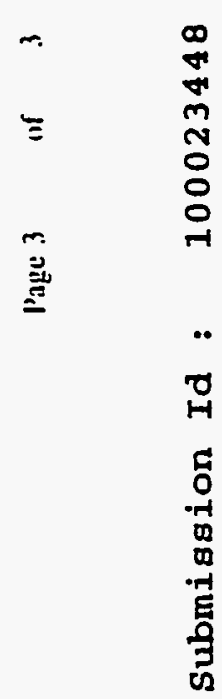

400000
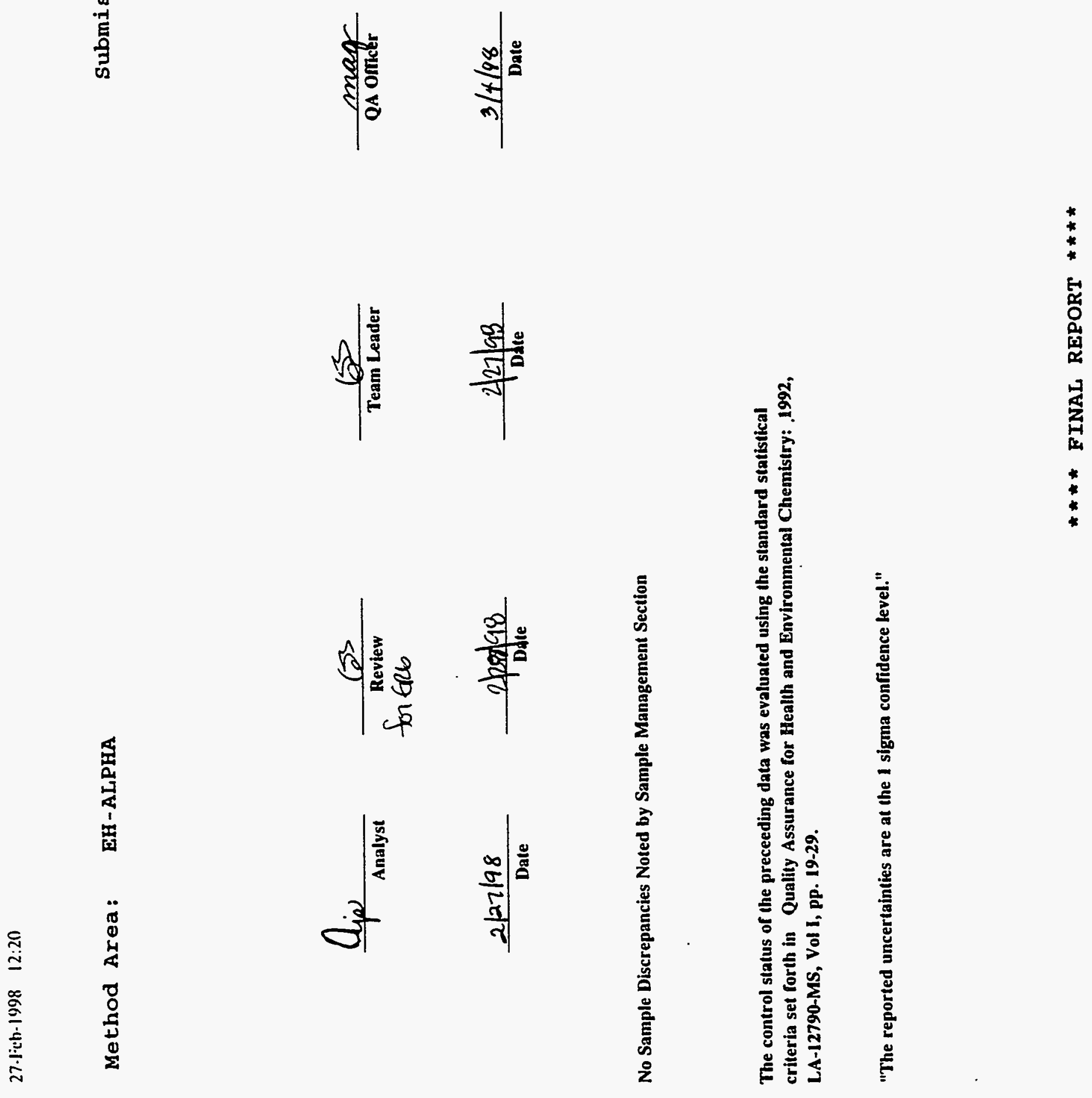
9
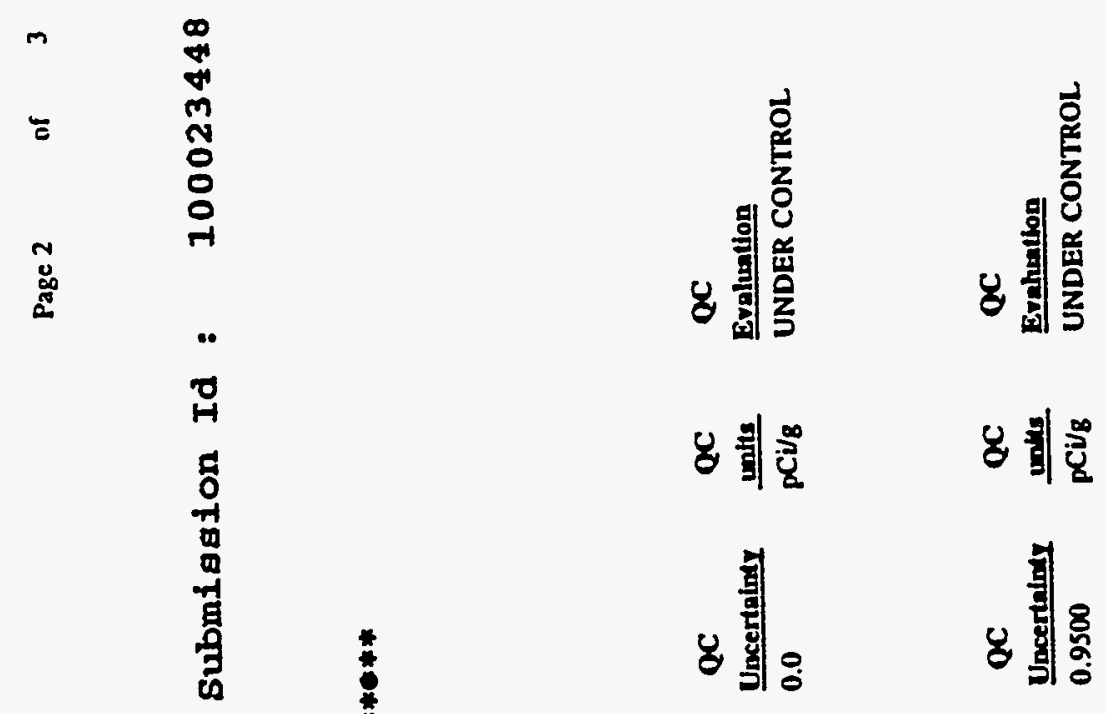

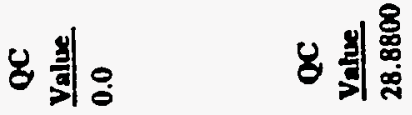
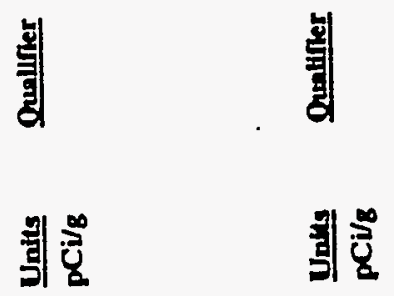

명
0
0
0
013

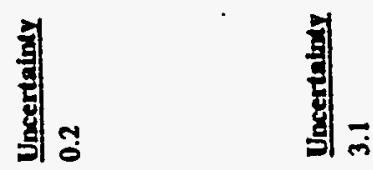

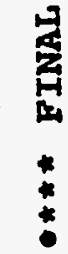

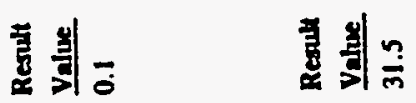

起

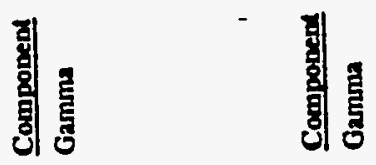

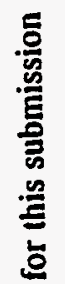

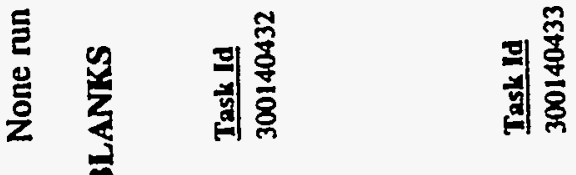

영

$\ddot{0} \ddot{0}$ 之

旁

总部

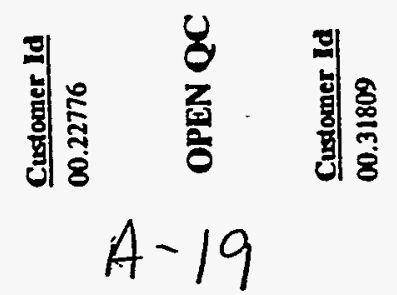



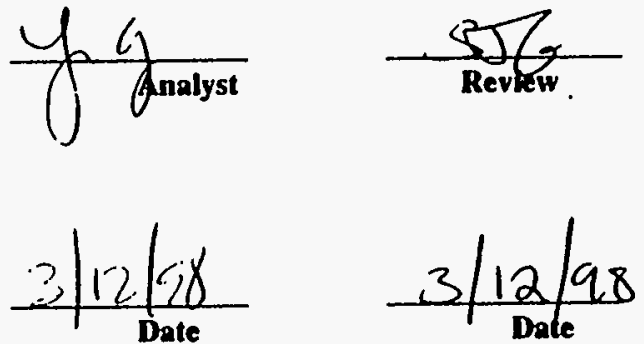

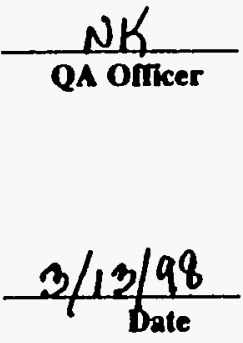

No Sample Discrepancies Noted by Sample Management Section

The control status of the preceeding data was evaluated using the standard statistical criteria set forth in Quality Assurance for Health and Environmental Chemistry: 1992, LA-12790-MS, Vol I, pp. 19-29.

"The reported uncertainties are at the 1 sigma confidence level." 